Transcontinentales

continentales

Sociétés, idéologies, système mondial

La transition chinoise

\title{
La Chine et ses campagnes
}

L'« édification de nouvelles campagnes socialistes » : un remède contre le malaise rural chinois?

\section{Sébastien Colin}

\section{(2) OpenEdition}

12 Journals

Édition électronique

URL : http://journals.openedition.org/transcontinentales/537

DOI : 10.4000/transcontinentales.537

ISBN : 978-2-8218-1112-6

ISSN : $1775-397 X$

\section{Éditeur}

Editions de la maison des sciences de l'homme

\section{Édition imprimée}

Date de publication : 31 décembre 2006

Pagination : 47-66

ISBN : 978-2-200-92170-5

ISSN : 1950-1684

\section{Référence électronique}

Sébastien Colin, «La Chine et ses campagnes », Transcontinentales [En ligne], 3 | 2006, mis en ligne le 12 octobre 2011, consulté le 10 décembre 2020. URL : http://journals.openedition.org/ transcontinentales/537 ; DOI : https://doi.org/10.4000/transcontinentales.537

Ce document a été généré automatiquement le 10 décembre 2020.

Tous droits réservés 


\title{
La Chine et ses campagnes
}

\author{
L'« édification de nouvelles campagnes socialistes » : un remède contre \\ le malaise rural chinois?
}

\section{Sébastien Colin}

1 Au début du mois d'octobre 2005, lors de la cinquième session plénière du XVI Comité central, le Parti communiste chinois a officiellement adopté le 11. plan quinquennal (2006-2010) au cours duquel il appelle à l'« édification de nouvelles campagnes socialistes (jianshe shehui zhuyi xin nongcun) ». Cette nouvelle politique à destination des campagnes, où vivent encore 750 millions de Chinois, a pour objectif principal de résoudre les différents maux qui accablent une grande partie du monde rural depuis maintenant près d'une quinzaine d'années. Après avoir placé les campagnes au centre des premières réformes, les autorités chinoises se sont ensuite penchées sur le développement des villes, reléguant les questions rurales au second plan. Les campagnes chinoises n'ont donc pas pleinement profité de la fulgurante ascension économique du pays durant la décennie 1990. Bien au contraire, cette période a vu s'accumuler divers maux, si douloureux pour les paysans chinois que des tensions de plus en plus palpables animent aujourd'hui certaines régions agricoles du pays. Les dernières jacqueries révélées par les médias occidentaux en 2005 et en 2006 en sont la preuve, mais elles ne sont que la partie émergée et médiatisée d'une colère encore timide et susceptible d'exploser à tout moment.

2 Après un bref rappel des réformes rurales de la fin des années 1970 et de leurs conséquences, dans un premier temps bénéfiques aux campagnes sur le plan socioéconomique, cet article décrira les principaux maux des campagnes chinoises, qui se sont accumulés au cours de la décennie 1990, et les remèdes prescrits par le régime chinois pour les guérir. Nous nous arrêterons donc en partie sur le contenu du programme des «nouvelles campagnes socialistes» et tenterons d'en fixer les limites. Même si l'absence de recul rend l'évaluation de cette politique très difficile, la place peu claire que prennent en son sein les questions de propriété foncière et de permis de résidence (hukou), qui sont pourtant deux problèmes centraux des campagnes chinoises et qui mériteraient en priorité des réformes, laisse pour l'instant douter de sa réelle efficacité. De ce fait, nous avons jugé utile de revenir sur la question du permis de 
résidence et du défi de l'exode rural : ce sont certes des problèmes qui touchent également les villes, mais ils symbolisent une certaine domination de la campagne par l'État, laquelle apparaît à bien des égards comme une des sources des difficultés que connaît aujourd'hui le monde rural chinois.

3 Cet article n'a pas pour objectif d'esquisser un portrait de l'agriculture chinoise. Sur ce point, nous renverrons le lecteur aux travaux de Claude Aubert, spécialiste reconnu de la question, dont certains des articles récents figurent en bibliographie. Disons simplement que la Chine a ceci de particulier d'avoir une agriculture dynamique, globalement autosuffisante, avec une part de plus en plus importante de cultures commerciales orientées vers l'exportation, dont les revenus profitent plus à la Chine industrielle et urbaine qu'à la Chine rurale encore marquée par endroits par les stigmates du sous-développement.

\section{À l'ombre des villes : âge d'or et crise sociale des campagnes chinoises}

4 En un peu plus d'un demi-siècle, les campagnes chinoises ont connu de nombreux bouleversements. La collectivisation, le Grand Bond en avant, la Révolution culturelle et la décollectivisation ont tour à tour modifié, certes à des degrés d'intensité différents, les modes et les conditions de vie des paysans chinois ainsi que l'organisation du monde rural. Pour autant, nous ne nous arrêterons ici que sur le dernier épisode, fruit des réformes de Deng Xiaoping, sans revenir sur les différentes politiques rurales de la Chine maoïste qui ont déjà été décrites et analysées par ailleurs 1 .

\section{Le temps des réformes : décollectivisation et industrialisation rurale}

5 Les réformes de la fin des années 1970 et de la première moitié des années 1980 ont été caractérisées par l'instauration de "systèmes de responsabilités » (zerenzhi) qui ont abouti à la généralisation des "contrats d'exploitation intégraux» (baogan daohu). Les premiers visaient à responsabiliser et à motiver les paysans en liant leur rémunération à la production. Les seconds ont été finalement passés avec les familles qui sont alors redevenues totalement responsables de leur exploitation et de leurs pertes et profits. Hormis le paiement de redevances collectives et le respect des livraisons obligatoires et forfaitaires (qui ne concernent plus que les céréales à partir de 1985) à l'État, à des prix fixés par lui, les ménages paysans ont la possibilité de diversifier leur production et de vendre leur surplus sur les marchés libres ${ }^{2}$.

6 L'établissement des "contrats d'exploitation intégraux» a dans le même temps nécessité entre chaque foyer paysan une division égalitaire des terres, sur lesquelles leur a été uniquement accordé un droit d'usage. Les terres sont restées la propriété du collectif, généralement représenté par les cantons (xiang) ou les villages ( $\underline{\text { cun }}$ ), qui sont progressivement redevenus les échelons de base du territoire rural chinois à la place des communes populaires, dont le démantèlement a été achevé à la fin de l'année 1984 3 .

7 Enfin, parallèlement à cette décollectivisation, les réformes ont aussi autorisé les usines de production et de réparation des anciennes communes populaires à fabriquer pour le 
marché intérieur chinois et pour l'exportation. Plus largement, dans l'objectif de créer des emplois non-agricoles et de favoriser un développement, elles ont encouragé les différentes autorités locales et les agriculteurs à utiliser leurs propres ressources (terre, main-d'œuvre, capital) pour établir des entreprises, appelées entreprises de bourgs et de cantons $(\mathrm{EBC})$ (xiangzhen qiye).

8 Ces différentes réformes ont incontestablement été bénéfiques aux campagnes chinoises. La libéralisation de l'agriculture et le développement des EBC ont permis aux ménages paysans d'augmenter leurs revenus. La décollectivisation s'est soldée par une augmentation spectaculaire de la production agricole et, sous l'impulsion des autorités, a permis une véritable spécialisation régionale, lançant ainsi l'agriculture chinoise sur la voie de la performance. Le développement des $E B C$ a quant à lui généré une industrialisation de la Chine rurale et a contribué à l'urbanisation des bourgs (zhen) et des villages. Les autorités locales ont en effet utilisé les revenus issus de ces entreprises pour développer différents types d'infrastructures (zones industrielles, ponts, routes, ports...) et réaménager leur territoire ${ }^{4}$. Surtout, ce développement des EBC a permis aux autorités chinoises d'apporter une première réponse à l'importante question de l'exode rural.

9 En effet, en générant un morcellement des terres agricoles, dont la superficie moyenne s'est avérée bien vite insuffisante pour faire travailler toute la famille, la décollectivisation a rapidement mis au jour un surplus de main-d'œuvre agricole prêt à se rendre en ville pour chercher un emploi. Face à ce défi, les EBC ont donc eu pour rôle d'embaucher prioritairement les populations locales afin d'absorber l'excédent de la main-d'œuvre rurale environnante. Les chiffres parlent d'eux-mêmes : les EBC ont créé annuellement plus de 12 millions d'emplois entre 1984 et 1988 puis 7,8 millions entre 1992 et 1996. Sur l'ensemble de ces deux périodes, ces emplois ont représenté plus de $90 \%$ des emplois non-agricoles présents dans les zones rurales. La plupart des paysans candidats au départ ont donc pu "quitter la terre sans quitter le canton " (li tu bu li xiang) et "entrer dans l'industrie sans entrer dans la ville " (jin chang bu jin cheng), soulageant, mais pour quelque temps seulement, les autorités chinoises.

On le voit, les réformes rurales de la fin des années 1970 et du début des années 1980 ont été très bénéfiques aux campagnes sur le plan socio-économique. Dès lors, comment expliquer le malaise actuel? Pourquoi les campagnes sont-elles présentées aujourd'hui comme les régions perdantes de l'ouverture et des réformes? Et ces situations, le développement comme la crise, sont-elles bien l'apanage de toutes les campagnes?

\section{Des campagnes délaissées et en difficulté}

11 Les aléas de la conjoncture économique ne peuvent suffire à expliquer le malaise des campagnes chinoises. Les crises céréalières du milieu des années 1980, puis de la fin des années 1990 et du début des années 2000, ont sans aucun doute posé quelques difficultés aux paysans les plus dépendants de cette production et ne disposant que de possibilités de diversification limitées. Et ce d'autant plus que le soutien des prix par l'État, à la fin des années 1990, s'est alors révélé impuissant à contrer la chute des prix et des productions. Paradoxalement, c'est la suppression du monopole en 2004 qui a permis au secteur céréalier de retrouver un équilibre relatif. De même, les difficultés rencontrées par les EBC à partir du milieu des années 1990 ont fortement amoindri leur 
rôle de palliatif économique. Après l'âge d'or des années 1984-1996, beaucoup d'entre elles ont été incapables de survivre sur un marché intérieur chinois devenu de plus en plus concurrentiel. Certaines des EBC travaillant essentiellement à l'exportation ont aussi subi les contrecoups de la crise asiatique. Près de 2,7 millions d'emplois par an ont ainsi été supprimés entre 1997 et 1999. Si la situation s'est sensiblement améliorée depuis, les EBC ne fournissent cependant plus autant d'emplois qu'auparavant. On assiste même à un plafonnement du marché du travail dans les entreprises rurales qui tournerait autour de 130 millions de travailleurs ${ }^{5}$.

En fait, les causes premières du malaise paysan sont surtout à chercher dans les orientations qui ont été données au développement au milieu des années 1980 puis au début des années 1990. Après avoir été privilégiés par les premières réformes, les campagnes et les paysans chinois ont alors été relégués au second plan, derrière l'industrie et les villes. Car, malgré de très nets progrès dans le domaine agricole, l'ensemble du système économique chinois était encore bien peu productif au milieu des années 1980. Les autorités ont donc décidé de ne plus limiter leurs réformes « au seul secteur rural et à quelques milliers d'entreprises ", mais de se pencher sur "l'ensemble du secteur urbain et les structures générales de l'économie chinoise " ${ }^{6}$. Cette orientation industrialo-urbaine a ensuite été largement confirmée lors de la relance des réformes par Deng Xiaoping en 1992. À bien des égards, l'ensemble de la décennie 1990 s'est caractérisé par un véritable abandon des campagnes.

Ce délaissement des campagnes au profit des villes est particulièrement visible dans l'évolution comparée des revenus ruraux et urbains. Certes, les seconds ont toujours été supérieurs aux premiers, mais on assiste depuis le milieu des années 1980 à un accroissement progressif de l'écart, alors même que les réformes de 1978 avaient justement permis un léger rééquilibrage (tableau ci-contre). En pourcentage du revenu urbain moyen, le revenu rural moyen est ainsi passé de $39 \%$ en 1978 à $54 \%$ en 1985, avant de s'effondrer et d'atteindre $36 \%$ en 2000 et $30 \%$ en 2005. Pourtant, le revenu moyen des ménages ruraux n'a jamais cessé d'augmenter. Sa croissance, en yuans constants, a cependant été moins rapide que celui des ménages urbains : en moyenne $4,2 \%$ par an, contre $6,7 \%$, entre 1985 et $2004{ }^{7}$.

14 La cote d'alerte en matière de disparités sociales semble atteinte : l'écart n'a jamais été aussi grand et demeure largement supérieur à ce qu'il était au sortir de la période maoïste. Bien sûr l'augmentation des revenus urbains est un immense progrès et mérite d'être saluée, mais elle n'est pas facile à accepter pour les paysans qui s'en sentent exclus. D'autant plus que la situation demeure dans les faits beaucoup plus inégalitaire que ne le laissent penser ces simples chiffres. Le fossé entre ruraux et urbains est en effet plus grand si l'on prend en compte les privilèges et services dont jouissent encore les populations des villes, notamment dans les domaines de la santé et de l'éducation, alors que les frais d'éducation et de santé pèsent lourdement sur les habitants des campagnes ${ }^{8}$. Jusqu'en 2006, s'ajoutait à cela le non moins lourd fardeau des taxes qui a longtemps grevé le revenu des ménages paysans, détériorant ainsi fortement leurs conditions de vies et leur pouvoir d'achat ${ }^{9}$. Les 750 millions de ruraux chinois, qui représentent $58 \%$ de la population totale, n'ont ainsi contribué qu'à 32,9\% des ventes au détail des produits de consommation en 2005. Ils sont également moins bien équipés en biens durables (télévision, réfrigérateur...) que ne le sont les ménages urbains. Les paysans chinois n'ont finalement pour eux que l'accès à la terre qui leur garantit fort heureusement la suffisance alimentaire et empêche une bonne partie 
d'entre eux de plonger dans -l'extrême pauvreté. Si les conditions de vie restent très précaires pour une très grande majorité de paysans chinois, il convient toutefois de signaler que la pauvreté absolue (moins de 1000 yuans par personne et par an selon les critères de la Banque mondiale) est circonscrite aux régions les plus naturellement déshéritées, notamment les régions montagneuses et arides de l'Ouest du pays ${ }^{10}$.

L'abandon des campagnes chinoises par les autorités centrales, manifeste dans le secteur de l'éducation, l'est aussi dans le domaine des retraites. Sont ainsi affectées deux catégories de populations, certes non-productives, mais qui représentent à la fois les anciennes et les futures forces vives du pays. Il n'y a toujours pas de système de retraite unifié dans les campagnes chinoises. Cette situation est d'autant plus préoccupante pour les personnes âgées qu'avec la mise en œuvre de la politique de l'enfant unique, lancée en 1979 (dans les faits deux enfants par ménage à la campagne), les agriculteurs ne pourront plus compter autant qu'autrefois sur leurs enfants pour assurer leurs vieux jours. La sécurité sociale est également quasi inexistante, ce qui oblige les paysans à payer eux-mêmes tous leurs frais médicaux, en constante augmentation.

16 Les campagnes chinoises font aussi face à d'importants problèmes dans le domaine éducatif. Les autorités centrales n'investissent pas assez dans l'enseignement primaire en zone rurale. Contrairement à la Corée du Sud et au Japon, le gouvernement chinois a laissé la question du financement de l'éducation dans les campagnes aux autorités des bourgs et des cantons qui, dans le contexte de décentralisation et de libéralisation des années 1990, n'ont pas su, pu ou voulu relever le défi. En effet, en dépit des nombreux prélèvements effectués sur les paysans, les communautés rurales ne sont plus en mesure de financer leurs écoles, soit parce que les ressources sont réellement insuffisantes, soit parce que les autorités locales définissent d'autres priorités (création d'entreprises rurales, construction d'infrastructures, etc.), voire dilapident une part de leur budget en achetant des nouvelles voitures pour les fonctionnaires locaux ou en organisant des banquets. Résultat, les infrastructures scolaires sont insuffisantes et les écoles rurales, sous-équipées, sont souvent dans -l'incapacité de verser les salaires des instituteurs qui ont tendance à quitter la campagne pour se rendre en ville. On assiste surtout à une redistribution géographique des écoles selon la logique de l'offre et de la demande. Les écoles ferment dès que le nombre d'élèves est jugé insuffisant pour financer leur fonctionnement, handicapant les régions en cours de dépeuplement et obligeant souvent les enfants qui -restent à faire de très longues distances pour être scolarisés. En fait, la généralisation de la scolarisation obligatoire dans les zones rurales, principal objectif du régime dans le secteur éducatif depuis 1986, n'est toujours pas réalisée. Officiellement, $76 \%$ des enfants sont scolarisés dans les campagnes, mais ce taux cache sans doute un fort taux d'abandon en cours d'année, qui reste toutefois difficile à évaluer ${ }^{11}$.

17 Les bras de fer que se livrent les communautés rurales et urbaines pour la terre et les ressources en eau, qui se concluent souvent par une victoire de la ville, sont encore des handicaps qui s'ajoutent à la morosité ambiante. Les questions de rétrocession des terres rurales à des fins de développement industriel et urbain sont au centre de nombreuses tensions, d'autant plus que le rapport de force demeure extrêmement déséquilibré. Les paysans ne peuvent plus faire grand-chose face à la puissance de la ville et sont bien souvent contraints d'accepter les rémunérations, de surcroît insuffisantes, offertes en échange de la réquisition de la terre. 
18 Notons enfin que les campagnes chinoises sont régulièrement frappées par des inondations, des typhons ou encore des périodes de sécheresse ${ }^{12}$, autant d'aléas climatiques qui sont bien souvent fatals aux plus fragiles et qui, année après année, accentuent la misère rurale. Certains villageois ne parviennent jamais à se remettre sur pied, quand ce ne sont pas des communautés entières, anéanties par l'épuisement des ressources consécutif à ces catastrophes récurrentes.

\section{Des campagnes divisées}

19 Ce préoccupant état des lieux des campagnes chinoises doit cependant être relativisé, ce qui n'enlève rien au défi qu'il pose. Il serait erroné de considérer cette situation comme homogène et d'omettre de la confronter aux disparités provinciales, qui conduisent à distinguer la Chine côtière, la Chine centrale et la Chine de l'Ouest. Les campagnes chinoises sont en effet très diverses et traversées par des inégalités de toutes sortes. On s'en tiendra ici à une analyse à l'échelle des provinces, mais il convient de garder à l'esprit que ces inégalités peuvent exister à toutes les échelles du territoire chinois.

20 Ainsi, les cartographies du nombre d'entreprises rurales dans les provinces chinoises et de la valeur de leur production, en 1994 - année où l'on compte le plus d'EBC en Chine et en 2002, montrent bien le fossé qui existe entre la Chine orientale, plus densément peuplée et ethniquement Han, et la Chine occidentale (cartes 1 et 2). Si les provinces encore très agricoles du centre de la Chine ont bien souvent un nombre d'EBC analogue, voire supérieur, à celui des provinces côtières (cartes 1), il n'en est pas de même de la productivité. Les entreprises rurales des provinces côtières ont une production dont la valeur est généralement supérieure à celles des provinces centrales les plus dynamiques (cartes 2). Or ces disparités -attirent d'autant plus l'attention qu'un des faits majeurs de ces dernières années a été justement «la montée en puissance des revenus non-agricoles dans les budgets des ménages paysans » : de $32 \%$ en 1985 à $54 \%$ en 2003 ; ils représenteraient, en termes monétaires nets, autour des deux tiers du budget total ${ }^{13}$. Or cette dépendance sous-entend que les inégalités de revenus au sein même de la Chine rurale seraient en partie le fruit de l'inégalité d'accès aux emplois non-agricoles offerts par les $\mathrm{EBC}$. La carte 3 , qui montre les très grandes inégalités de revenus ruraux entre les provinces chinoises, tend largement à confirmer cette hypothèse. 
Carte 1

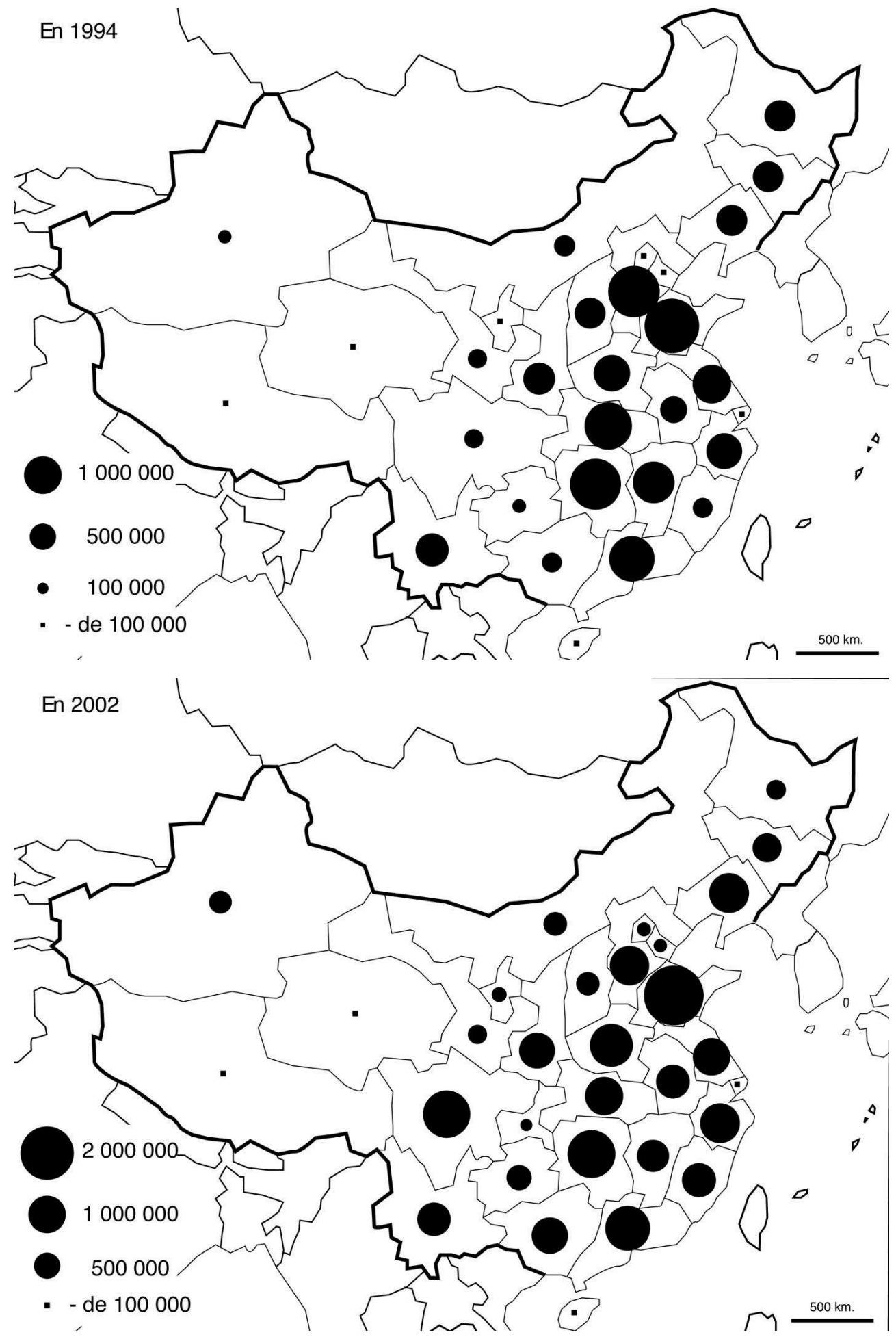

Nombre d'entreprises chinoises dans les provinces chinoises en 1994 et en 2002 
Carte 2

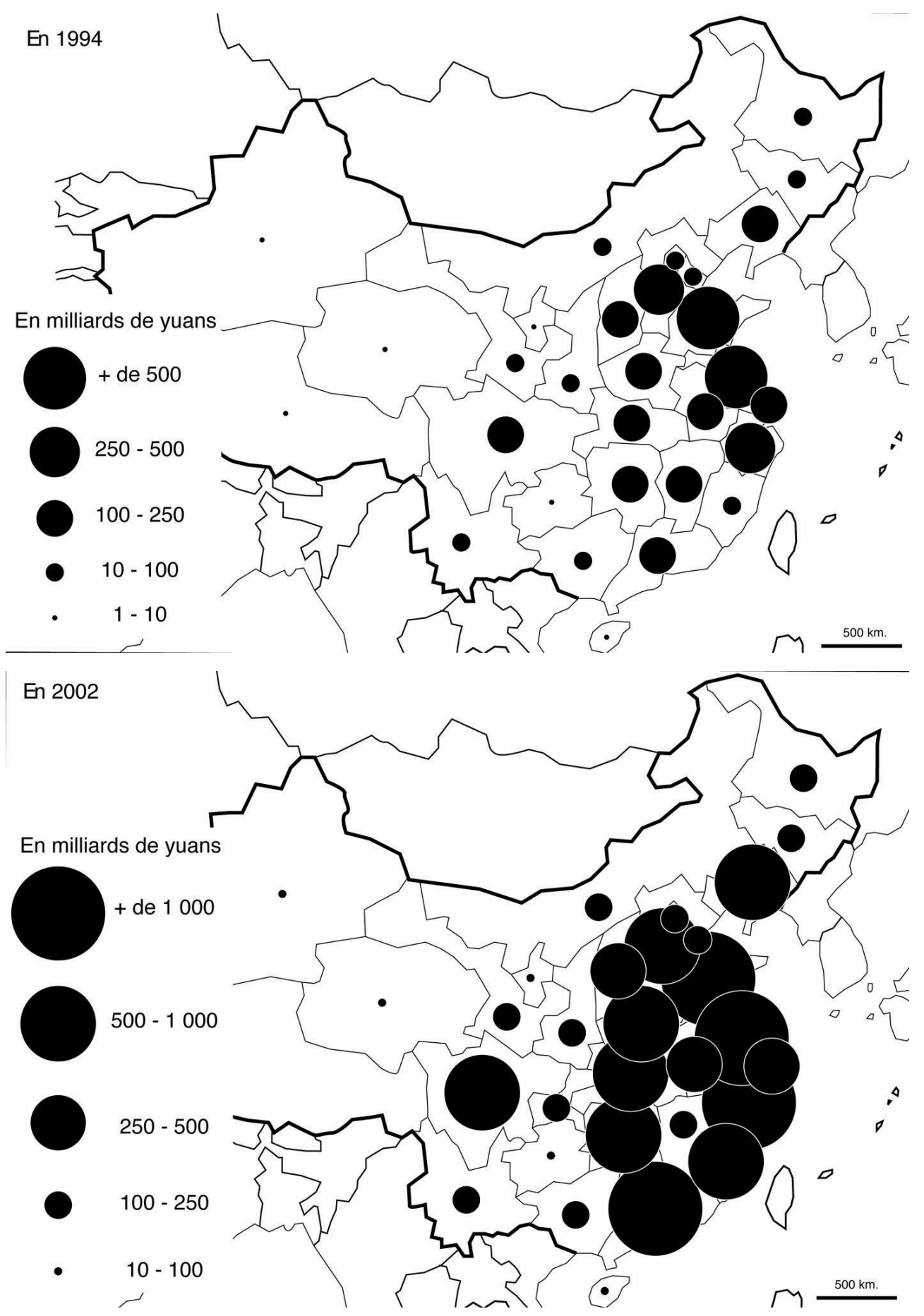

Valeur de la production des entreprises rurales dans les provinces chinoises en 1994 et en 2002. 


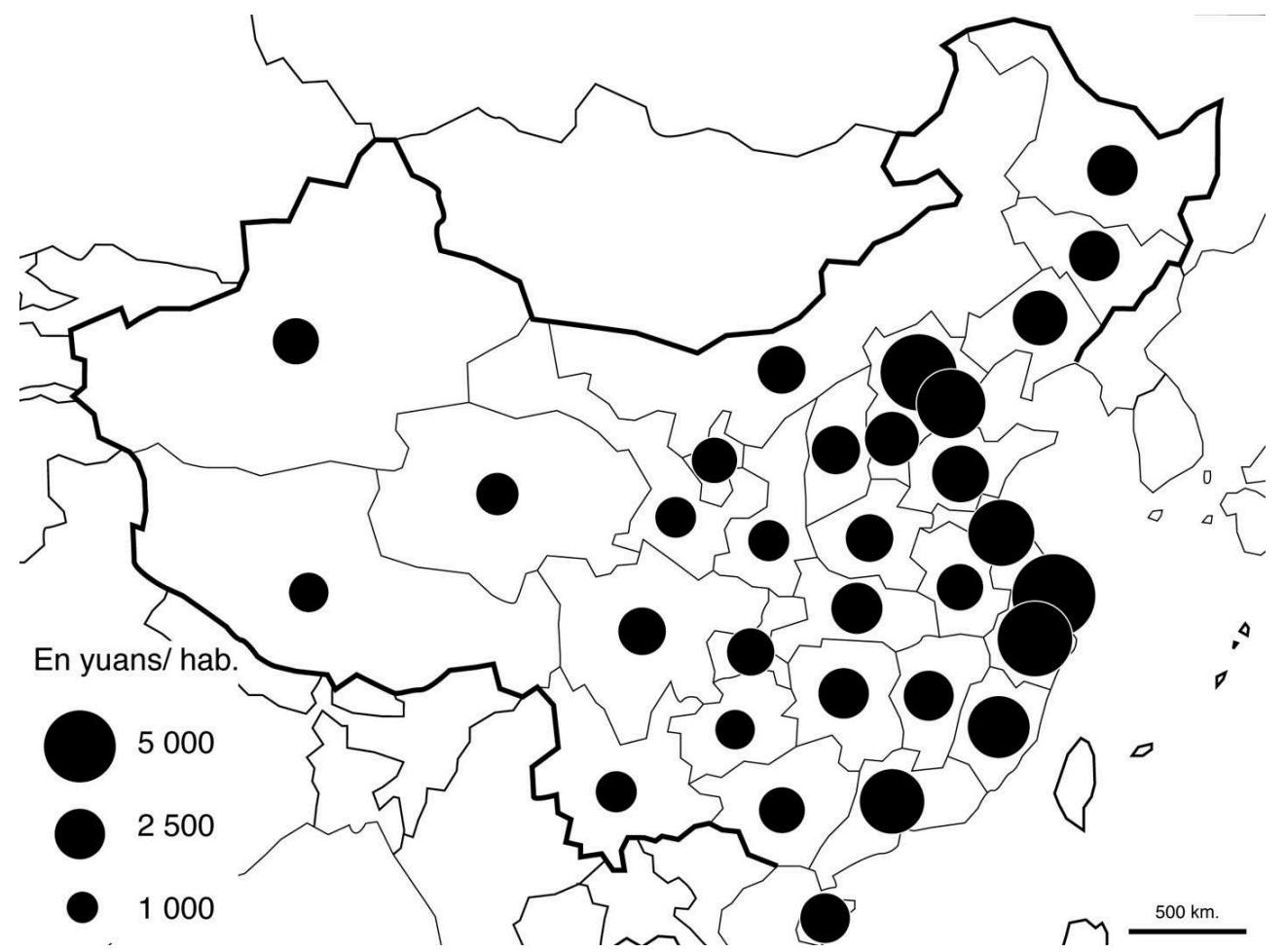

Revenu par tête des populations rurales dans les provinces chinoises en 2003

\section{Des campagnes en colère}

Les écarts de revenus, le fardeau des charges, l'absence de protection sociale et la réquisition sous-indemnisée des terres agricoles sont autant de facteurs qui, à terme, ont fini par excéder les paysans. Souvent aggravés par la corruption, voire la violence, de certains cadres locaux, ils sont régulièrement à l'origine d'émeutes, qui ne sont certes pas nouvelles mais qui ont eu tendance à se multiplier ces dix dernières années. Le pouvoir aurait ainsi recensé autour de 86000 «incidents» en 2005. Aucune information supplémentaire n'ayant été divulguée concernant ces chiffres, il n'est malheureusement pas possible d'en établir une géographie qui serait pourtant riche d'enseignements. D'après les faits recensés par les médias, il semble toutefois que ces émeutes aient surtout lieu dans les campagnes périurbaines, là où se cristallisent les tensions concernant la terre ou l'eau entre la ville et la campagne et où les disparités de revenus sont les plus visibles. On ne reviendra pas ici sur les événements, dont certains des plus récents, par les violences qu'ils ont mises au jour, ont trouvé de larges échos dans les médias occidentaux ${ }^{14}$. On s'interrogera plutôt sur leurs significations politiques.

Ces émeutes forment en effet avec les pétitions un binôme, appelé shangfang naoshi (pétitions et troubles), qui devient pour les masses paysannes « une méthode normale et utile de communication avec les instances supérieures (municipalité, préfecture, province, gouvernement central) de l'État et du Parti » afin de "défendre leurs intérêts ». Son émergence tend à souligner l'inefficacité du passage par les "canaux institutionnels et légaux » destinés à enregistrer les plaintes au niveau local auprès des « instances les plus immédiates ». Les quelques paysans qui s'y sont essayés jugent en 
effet cette démarche peu utile. La plupart des requêtes ne trouvent aucune suite, bien souvent bloquées par les cadres locaux qui y voient une critique à leur encontre d'autant plus dangereuse que c'est en fonction du nombre de plaintes que leur travail est évalué par les instances supérieures. En fait, l'utilisation croissante du binôme pétition-émeute, malgré les risques qu'il représente, témoigne du fossé qui s'est creusé entre la base et les cadres locaux. Ces derniers sont bien souvent perçus par les paysans comme des "pillards» (lüedozhe), complices des expropriateurs urbains et des exacteurs lors des répressions, et peu soucieux de défendre leurs intérêts. En retour, les cadres considéreraient les paysans plaignants comme "des gens rusés et violents" (diaomin, baomin) et leurs pétitions et émeutes comme "des phénomènes anormaux " (bu zhengdang), voire « oppositionnels » (dikang), justifiant amplement la répression ${ }^{15}$.

Il ne fait aucun doute que cette mauvaise réputation des cadres, en tant que représentants locaux du Parti et de l'État, nuit gravement à la légitimité du régime. Cette situation inquiète d'ailleurs fortement les autorités centrales qui n'hésitent pas à renvoyer la responsabilité des émeutes sur les cadres locaux en les accusant d'incompétence voire de corruption. Néanmoins, l'ambiguïté et le flou qui marquent le rôle réel des autorités centrales dans la répression des émeutes montrent qu'elles ne sont pas prêtes à tolérer ni à excuser ces mouvements paysans.

Ces derniers peuvent-ils représenter une réelle menace pour le régime? La réponse à cette question semble pour l'instant négative. Géographiquement éclatées, les revendications paysannes sont surtout d'ordre social et n'intègrent aucune revendication politique : l'heure est à l'amélioration des conditions de vie et non au souhait d'établir une démocratie. Les paysans demandent juste un peu plus d'attention de la part du régime et, surtout, sa protection contre les assauts prédateurs du monde industriel et urbain. En ce sens, les nombreuses pétitions envoyées aux autorités centrales, en prenant bien soin de contourner l'intermédiaire des cadres locaux, montrent que les paysans n'ont pas encore totalement perdu confiance dans les capacités d'action du régime. En revanche la situation peut changer. Les paysans pourraient très bien constituer une force importante de contestation pour peu qu'ils soient structurés ou guidés par quelques membres plus politisés de la société chinoise. Ils seraient alors d'autant plus menaçants qu'ils pourraient s'unir avec d'autres mouvements sociaux, pour l'instant également isolés, comme ceux des chômeurs ou des migrants ruraux. On comprend mieux dès lors pourquoi la question des campagnes est redevenue une priorité du régime depuis le début des années 2000.

\section{Vers de « nouvelles campagnes socialistes »?}

\section{Le retour des campagnes dans les préoccupations du régime}

En 2004 et en 2005, le Comité central du parti communiste a placé les paysans, les campagnes et l'agriculture au centre de ses préoccupations en leur consacrant notamment son « Document $\mathrm{n}^{\circ} 1$ » qui marque généralement les priorités politiques de l'année à venir. Les objectifs étaient, pour 2004, d'augmenter les revenus paysans et, pour 2005, de renforcer la productivité agricole. Pour ce qui est de l'augmentation des revenus, les autorités n'ont guère eu d'autres choix que de travailler à la réduction puis à la suppression des charges fiscales. C'est ainsi que l'ensemble des taxes et autres prélèvements agricoles et para-agricoles sont totalement abolis depuis janvier 2006. 
Pour ce qui est de la productivité agricole, l'objectif est surtout de faire de la Chine une grande puissance exportatrice dans l'agroalimentaire. La question de l'autosuffisance en céréales, officiellement évoquée pour justifier cette politique, n'aurait quant à elle guère de sens puisque la Chine, sauf problème politique ou climatique majeur, subviendrait déjà à ses propres besoins ${ }^{16}$.

Un nouvel élan a ensuite été donné en octobre 2005 avec l'intégration par le gouvernement central du programme «d'édification de nouvelles campagnes socialistes " (jianshe shehui zhuyi xin nongcun) dans le 11• plan quinquennal (2006-2010). Après avoir officiellement reconnu les différents maux qui touchent les zones rurales, le Premier ministre Wen Jiabao a annoncé que son gouvernement leur accorderait désormais plus d'attention. Les objectifs sont nombreux et très ambitieux. Les autorités prévoient ainsi d'instaurer la gratuité de l'école pour tous les paysans en 2007 et un régime public d'assurance-santé dans $80 \%$ des régions rurales d'ici la fin de l'année 2008. À ces promesses vient s'ajouter la volonté de relancer les travaux d'infrastructures hors des grandes cités. Pour mener à bien ces projets, une enveloppe de 339,7 milliards de yuans (environ 34 milliards d'euros) a déjà été libérée pour l'année 2006. Par la suite, 2 à 3 points du PIB devraient être consacrés chaque année à cette politique. Les autorités misent également sur une solidarité entre la ville et la campagne et entre l'industrie et l'agriculture. Selon elles, les villes et les industries auraient effectivement tout à gagner de la réussite de cette politique qui devrait stimuler la demande intérieure et la consommation dans les zones rurales, permettant ainsi d'éviter une accumulation trop importante des surcapacités industrielles et de dégonfler les excédents commerciaux, en bref, d'apporter des solutions aux déséquilibres de la croissance. Enfin, dans l'objectif de réglementer et d'éviter les excès des réquisitions, un dernier volet promet de revoir les procédés d'acquisition des terres rurales, sans pour autant annoncer les modalités qui seront mises en place pour y parvenir. On se doute toutefois que, sur cette question, le débat fait rage entre les partisans du maintien du système collectif et ceux de la libéralisation totale. Pour ces derniers, la privatisation du foncier aurait l'avantage de faciliter la concentration des terres, passage obligé, selon eux, vers une réelle modernisation de l'agriculture. Pour autant, il n'est pas sûr que cette solution satisfasse vraiment les paysans. Après tout, les contrats actuels, même s'ils ne protègent en rien les paysans contre les réquisitions, ont toutefois l'avantage de garantir l'accès à la terre à chaque famille, représentant ainsi un moyen de survie pour les plus pauvres ${ }^{17}$.

L'autre point, qui parait central pour régler une partie des problèmes ruraux mais qui n'est pas pleinement abordé dans les objectifs de cette nouvelle politique, demeure le grand défi de l'exode rural. Car si le développement des EBC a permis dans un premier temps de contrôler et de canaliser l'exode rural vers les petites villes, la restructuration et la disparition de certaines de ces entreprises rurales durant la seconde moitié des années 1990, ainsi que l'accroissement des inégalités au sein du monde rural, ont fini par reposer, et de manière beaucoup plus aiguë, la question de la main-d'œuvre inoccupée de la campagne, bien décidée cette fois à " quitter la terre et le canton » (li tu you li xiang) pour aller chercher du travail dans les villes et leurs zones périurbaines.

\section{Le grand défi de l'exode rural et la réforme du hukou}

S'il est une manifestation incontournable du développement, l'exode rural constitue en Chine, compte tenu du poids démographique des populations rurales, un enjeu de taille. 
Il est composé de deux types de migration. Le premier est la migration formelle (qianyi), qui est approuvée officiellement et parfois planifiée par l'État, notamment lors de la relocalisation volontaire de personnes dans le cadre d'une politique de développement local, à la suite d'une catastrophe naturelle, en réponse à la construction d'un gros ouvrage, ou encore en raison d'une restructuration urbaine. Le second type est une migration informelle et spontanée qui est de très loin la plus problématique pour les autorités. Les populations qui composent ses flux sont -qualifiées de "populations flottantes" (liudong renkou), "d'ouvriers-paysans" (nong mingong ou mingong) ou encore de "populations venues d'ailleurs » (wailai renkou). Cette migration informelle a rapidement attiré l'attention des chercheurs.

Des études permettent aujourd'hui de dresser un portrait-type du migrant (jeune, éducation moyenne à bonne, travaillant dans les villes développées au moins six mois dans l'année et migrant grâce au réseau de parenté et ou du village), mais l'ampleur de ces migrations est toujours difficile à évaluer avec exactitude. Le nombre de migrants aurait été de 100 millions en l'an 2000, serait passé depuis à 150 millions, et augmenterait actuellement de 7 à 8 millions par an ${ }^{18}$. Si la majorité d'entre eux travaillent encore dans les EBC et s'installent le plus souvent dans des villes de leur province d'origine, les migrations rurales de longue distance se sont néanmoins considérablement accrues ces dernières années. Sans surprise, ces flux interprovinciaux vont principalement des provinces centrales (Sichuan, Anhui, Jiangxi, Hunan, Hebei, Henan) vers les villes et aires périurbaines des provinces côtières (Shanghai, Pékin, Zhejiang, Tianjin, Fujian, Guangdong...). La plupart des migrants se dirigeant vers ces villes sont des saisonniers qui cherchent surtout à augmenter rapidement leurs revenus; ils y gagneraient en moyenne deux fois le salaire moyen d'un paysan. En fait, le moteur de ces migrations rurales réside dans la conjugaison de trois facteurs : les inégalités de revenus entre les villes et les campagnes, le surplus de la main-d'œuvre agricole, et l'existence de nombreuses opportunités d'emplois (bien souvent des travaux pénibles dans les secteurs manufacturiers, du bâtiment et des services) dans les villes en fort développement ${ }^{19}$.

Mais les villes ne se sont pas encore complètement ouvertes à ces flux migratoires originaires des campagnes, en raison notamment de la permanence du système du permis de résidence (hukou). Ce système, rappelons-le, fut une des principales mesures prises par les autorités communistes à la fin des années 1950 pour restreindre la croissance urbaine. En 1958, l'Assemblée nationale populaire déclara que chaque foyer chinois devait disposer d'un livret d'enregistrement permanent (ou livret de famille hukou bu). Ce système distinguait les populations qui disposaient d'une résidence urbaine (chengshi hukou) de celles qui vivaient en milieu rural (nongcun hukou). L'objectif était d'ancrer les populations à leur lieu de naissance et d'empêcher les migrations non autorisées des campagnes vers les villes ${ }^{20}$. Le système du hukou était complété par un dispositif d'enregistrement des emplois. Chaque personne en âge de travailler avait ainsi son livret d'identité et de travail (gongzuo zheng) qui le rattachait à son unité de production (danwei) industrielle ou agricole. Enfin, aux hukou et gongzuo zheng s'ajoutait en ville un système de rationnement. Il permettait aux populations urbaines munies du permis de résidence urbaine (chengshi hukou) de venir s'approvisionner à bas prix en différents biens de consommation parmi lesquels figuraient les grains et le fioul, deux denrées indispensables à la vie quotidienne. Le changement de hukou ne pouvait se faire que par un transfert officiel. On en comptait trois types : le recrutement par une 
entreprise urbaine (zhao gong); l'embauche ou l'inscription dans un établissement éducatif de haut niveau (zhao sheng); et la promotion à un poste administratif (zhao gan). La mise en place de ce système n'a certes pas complètement éliminé la formation de flux migratoires spontanés des campagnes vers les villes, mais elle les a bien limitées. Jusqu'à la fin des années 1970, ceux-ci étaient majoritairement contrôlés par les autorités, notamment dans le cas de campagne de recrutement de main-d'œuvre rurale pour les unités urbaines de travail.

Cette politique de restriction des migrations a toutefois perdu une partie de son sens avec les réformes économiques. En fait, depuis 1979, les autorités chinoises n'ont cessé d'alterner, vis-à-vis de l'exode rural, prohibition, autorisation, restriction, régulation et encouragement. Dans leur travail sur les migrations rurales, Huang Ping et Frank N. Pieke ont mis en avant quatre grandes périodes caractérisant chacune l'une ou plusieurs de ces alternatives ${ }^{21}$. Nous ne nous arrêterons ici que sur les premiers assouplissements qui datent du milieu des années 1980. Leur objectif était surtout de limiter les migrations à des déplacements de proximité et d'éviter ainsi des migrations de longue distance à destination des grandes villes côtières. En 1984, l'État, dans un document officiel, autorisait ainsi les migrations saisonnières de paysans ; ils pouvaient adopter une occupation mobile comme le transport et le commerce et s'installer de manière permanente dans les bourgs pour travailler, à condition toutefois de s'arranger eux-mêmes pour leur ration de grains et de ne pas se rendre dans les boutiques aux prix subventionnés par l'État, réservées aux habitants du bourg; par ailleurs, les migrants ne pouvaient bénéficier d'aucune subvention financière et d'aucune allocation chômage. Dans le même temps, les autorités chinoises encouragèrent le développement des $\mathrm{EBC}$, dont l'un des buts, on l'a vu, était de limiter l'exode rural. En 1985, le ministère de la Sécurité publique assouplit un peu plus le système en mettant en place le hukou temporaire (zhanzhu hukou) ainsi qu'une "carte d'identification et de résidence " (jumin shenfen zheng), fournie à tous les citoyens de plus de seize ans. Cette dernière peut encore être utilisée pour chercher un emploi, s'enregistrer dans un hôtel, et surtout -obtenir le hukou temporaire.

Si ces premières mesures ont incontestablement permis une plus grande liberté de circulation, elles n'ont toutefois pas éliminé les véritables tares du hukou qui est resté un terrible outil de discrimination à l'encontre des migrants en zone urbaine. Il leur ôte l'accès aux privilèges urbains (gratuité des soins médicaux et du système éducatif, attribution d'un logement), dont certains tendent toutefois à disparaitre avec les réformes ${ }^{22}$, et continue d'interdire la venue de leur famille et leur installation à long terme. D'autres restrictions comprennent les entraves à l'entrée sur le marché du travail, ainsi que diverses taxes et dépenses additionnelles liées au statut de migrants (frais de lutte contre le crime, de construction et de gestion urbaine, ou même de planning familial pour les femmes). La majorité des migrants n'ont pas de couverture médicale et ne sont pas protégés en cas d'accident du travail. Les frais d'éducations restent prohibitifs en raison des frais de compensation liés à -l'absence de permis de résidence urbaine (chengshi hukou). Les mingong ont bien tenté de s'adapter en créant leurs propres écoles, mais celles-ci ont immédiatement été qualifiées d'illégales par les autorités centrales. Même si certains réussissent, les migrants ruraux sont en général en bas de l'échelle sociale et constituent une part importante des populations pauvres des villes. 
Sans être complètement intégrées aux objectifs de la politique "d'édification des nouvelles campagnes socialistes", ces questions des travailleurs migrants et du système du hukou ont toutefois été prises en considération par le régime au tournant des années 2000. Après une réforme expérimentale, menée en grande partie dans la province côtière $\mathrm{du}$ Zhejiang, les autorités centrales ont finalement décidé, le $1^{\mathrm{er}}$ octobre 2001, de supprimer officiellement le hukou dans la vingtaine de milliers de petites villes que compte le territoire chinois, sans modifier le dispositif pour les grandes métropoles. Au début de l'année 2002, le Conseil des affaires d'État a publié un document dans lequel il reconnaissait que les migrations du surplus de la maind'œuvre rurale vers les villes étaient une conséquence normale du développement économique et de la libéralisation du marché. Il définissait ces travailleurs migrants non plus comme des paysans mais comme des membres de la classe ouvrière qui contribuent activement au développement industriel et urbain depuis le début des années 1980. De fait, il condamnait les politiques restrictives infligées aux migrants et appelait à réfléchir à de nouvelles directives plus équitables visant à offrir un meilleur service à ces populations. Une partie de ces directives furent publiées par le Conseil des affaires d'État en janvier 2003. Le document fait d'abord la synthèse des problèmes rencontrés par les migrants ruraux dans les villes (accès limité à l'emploi, absence de protection juridique et sociale, long délai des paiements, frais arbitraires), avant d'énumérer un certain nombre d'assouplissements ${ }^{23}$, sans pour autant prôner une suppression pure et simple du hukou.

Cette nouvelle politique des autorités centrales va de pair avec la nouvelle politique rurale mais aussi avec une nouvelle politique urbaine, qui consiste à encourager l'urbanisation. Elle reflète également le débat que suscitent les questions du hukou et des migrations rurales au sein de la société chinoise. Depuis le milieu des années 1990, de plus en plus de voix réformatrices s'élèvent en effet pour demander une circulation plus rationnelle des individus. Selon elles, les différentes inégalités territoriales qui touchent le pays rendent très urgent le processus de réforme du permis de résidence. Le transfert de la main-d'œuvre paysanne disponible et excédentaire des campagnes vers les villes permettrait de dynamiser l'économie rurale en rendant possibles de nouvelles concentrations des terres tout en offrant de la main-d'œuvre supplémentaire pour le développement des villes. Pour ces partisans de la réforme, il est désormais primordial de supprimer l'association entre lieu de résidence obligatoire et statut économique et politique, ainsi que de ne plus soumettre à des conditions de résidences l'obtention de privilèges réservés aux citadins en matière d'éducation, de logement, de protection et d'assistance sociale. Ce point de vue continue toutefois de se heurter à celui des conservateurs. Pour beaucoup de dirigeants, un afflux massif et aveugle de la population rurale en milieu urbain serait un facteur de déstabilisation sociale; il entraînerait la création de quartiers pauvres, accélérerait la fuite des compétences des petites et moyennes villes vers les grandes ou encore creuserait le fossé déjà important entre un Est développé et un Ouest défavorisé. Pour eux, le système du hukou reste un outil important qui permet à l'État de réguler la taille de la population urbaine suivant ses propres intérêts et de maintenir le contrôle social.

35 Cette confrontation permanente risque sans doute de rendre difficile la pleine application de la réforme et, surtout, son élargissement aux grandes villes du pays. La réforme est de plus compliquée par deux préoccupations majeures du régime: la permanence d'un taux de chômage important dans les villes, particulièrement chez les 
jeunes diplômés et les ouvriers des anciennes entreprises d'État, et le financement de la couverture sociale des citadins, qui lui coûte déjà trop cher. Or les migrants ruraux continuent d'être perçus comme des concurrents sur le marché du travail et leur pleine insertion dans le monde urbain accentuerait le nombre des bénéficiaires potentiels de cette couverture sociale. La mise en place de la réforme est également entravée par la décentralisation administrative. Ainsi, il arrive que des autorités provinciales ou municipales prennent certaines libertés en n'appliquant que quelques directives ou en promulguant leur propre réforme à l'échelle de la province et de la municipalité ${ }^{24}$. Signe de la difficulté de sa mise en place ou réelle motivation du régime, la parution future d'un projet de loi sur le hukou, qui devrait instituer un nouveau système plus adapté aux mouvements migratoires de la société et garantir les droits de liberté et d'égalité pour tous les citoyens, a été annoncée en février 2005 par le ministère de la Sécurité publique. Malgré l'espoir que cette énième annonce a provoqué dans l'opinion publique, elle continue de susciter un grand nombre d'interrogations parmi les spécialistes.

\section{Des campagnes toujours dominées}

Malgré les réformes de la fin des années 1970 et du début des années 1980, les campagnes chinoises apparaissent toujours comme des espaces dominés par l'État et par la ville. Certes, en abolissant les taxes agricoles au début de l'année 2006, ce qui reste pour l'instant la mesure phare de la nouvelle politique «d'édification des nouvelles campagnes socialistes ", les autorités centrales ont voulu relâcher un peu cette domination. Le fardeau des taxes et des retenues collectives a en effet longtemps grevé le budget des paysans et a été à l'origine, au côté des expropriations de terres agricoles, de la plupart des émeutes ou jacqueries régulièrement rapportées par les médias. Rien n'assure toutefois que cette abolition fera disparaître à jamais la réalité de ce fardeau. La suppression des taxes agricoles constitue en effet une importante perte de revenus pour les districts et cantons, dont on imagine mal qu'ils puissent réduire d'autant leurs dépenses; elle nécessite donc un transfert financier du Centre et des provinces vers les campagnes. Ce dernier, qui doit s'élever à 103 milliards de yuans en 2006, demeurerait néanmoins très insuffisant et l'on pourrait "assister à une multiplication de taxes illégales de la part des gouvernements locaux, officiellement pour boucler leur budget, mais qui risque de laisser la porte ouverte à tous les abus possibles ${ }^{25} \%$.

La domination de l'État sur les campagnes chinoises se caractérise surtout par le comportement des cadres locaux qui y ont bien souvent tout pouvoir. Leur puissance s'illustre particulièrement lors des questions de réquisition des terres rurales, qui se règlent bien souvent de manière unilatérale, avec des méthodes parfois douteuses et face auxquelles les paysans n'ont aucun mot à dire. Si les autorités centrales ne manquent pas de critiquer certains de ces abus, les cadres locaux ne restent pas moins les représentants de l'État central aux yeux des paysans et tout comportement négatif de leur part entraîne donc de facto un discrédit sur l'État. Dans certaines campagnes, le fossé tend à se creuser entre les cadres et les paysans qui -menacent leur stabilité ${ }^{26}$. En fait, la toute-puissance de certains cadres locaux renvoie à la question, fondamentale en Chine, des rapports entre le Centre et le local et du difficile contrôle que le premier exerce sur le second. 

seconde moitié des années 1990 n'a aucunement éliminé ce rapport dominant/ dominé. D'après la loi de 1998, les comités de villageois sont en effet toujours sous la responsabilité de la branche locale du Parti. S'il est incontestable que les paysans ont théoriquement un pouvoir d'expression et de participation supérieur à ce qu'il était avant les réformes, la marge de manœuvre de ces comités demeure dans les faits très limitée et la décision finale est rarement de leur ressort ${ }^{27}$. De même la réforme quinquennale des tribunaux populaires, dont les grandes lignes ont été promulguées le 20 octobre 1999 et dont l'objectif est de trouver une nouvelle manière d'améliorer la gouvernance dans les campagnes, ne porte que sur les modalités de jugement, mais pas sur la dépendance envers le Parti, donc envers l'État ${ }^{28}$. Dans les faits, on a constaté que les élections des comités de villageois étaient surtout considérées comme des réponses à des crises locales, un moyen de regagner la confiance et le soutien des paysans en les invitant à participer à la vie politique du village et du canton, mais que leur organisation avait tendance à disparaître aussitôt la stabilité recouvrée

29. La réussite et le maintien d'une organisation régulière de ces élections dépendent plutôt du niveau de développement économique du village ou du canton. Certaines études ont en effet montré que là où la situation économique était prospère, les relations entre les cadres et les paysans s'en retrouvaient harmonisées, accentuant ainsi l'intérêt que les premiers pouvaient avoir à organiser ce type d'élections et les seconds à y participer ${ }^{30}$.

Mais pour parvenir à ce développement économique, la résolution de multiples problèmes pris de manière isolée et l'injection de subventions ne suffiront peut-être pas. Avant tout, les autorités chinoises devront définitivement régler des problèmes de fond comme le permis de résidence, la propriété et la gestion des terres rurales, qui sont les réels symboles de la domination des campagnes, ceux qui font d'elles des actrices du développement économique chinois tout en leur en refusant les fruits. À bien des égards, comme le laisse peut-être présager cette nouvelle politique "d'édification des nouvelles campagnes socialistes", les autorités centrales doivent aujourd'hui aborder les défis du pays de manière transversale et non plus de manière sectorisée (régions côtières/ arrière-pays, ville/ campagne...) comme elles l'ont souvent fait jusqu'à maintenant. Surtout, elles devront prendre à bras le corps l'épineuse question de gouvernance dans les campagnes chinoises : en somme, libérer les paysans du joug des cadres locaux et mieux contrôler ces derniers, sans pour autant remettre totalement en cause le processus de décentralisation. On le voit, ce défi est énorme, sans doute parce qu'il renvoie directement à une réforme politique du pays que le Parti refuse toujours d'aborder. Or il semble bien qu'il soit au centre de la plupart des problèmes que rencontre aujourd'hui le monde rural chinois. 


\section{BIBLIOGRAPHIE}

- AUBERT Claude (a), « Le devenir de l'économie paysanne en Chine », Revue Tiers-Monde, $\mathrm{n}^{\circ}$ 183, juillet-septembre 2005, p. 491-515, disponible en ligne : http://www.ivry.inra. fr/mona/ publications_chercheurs/Textes-Publis/Aubert-TMChine.pdf.

- AUBERT Claude (b), « Politiques agricoles chinoises : la porte étroite », in L'économie mondiale 2006, Paris, CEPI et La Découverte, coll. « Repères », 2005, p. 69-83, disponible en ligne : http:// www.ivry.inra.fr/mona/ publications_chercheurs/Textes-Publis/ Aubert-EcoMond06.pdf.

- AUBERT Claude, Ying CHENG et Xiande LI, «Éthique et économie paysanne en Chine ", in Marc DUFUMIER (dir.), Un agronome dans son siècle, actualité de René Dumont, Paris, Karthala, 2002, p. $131-138$

- BERGÈre Marie-Claire, La Chine de 1949 à nos jours, Paris, Armand Colin, 2000, 382 p.

- CHEN Jie, « Sociopolitical Attitudes of the Masses and Leaders in the Chinese Village : Attitude Congruence and Constraint », Journal of Contemporary China, 14(44), août 2005, p. 445-464.

- CHENG Weimin, « Some Thoughts on the Subjectivity of Villager's Self-government », Social Sciences in China, hiver 2005, p. 26-30.

- DING Wei, «Réforme des tribunaux de base et application de la loi dans la Chine rurale. Le cas de l'arrondissement de Weicheng, ville de Xianyang, province du Shaanxi », Perspectives chinoises, $\mathrm{n}^{\circ} 90$, juillet-août 2005, p. 45 et 47-48.

- DUMONT René, Révolution dans les campagnes chinoises, Paris, Le Seuil, 1957, 463 p.

- DUMONT René, La Chine surpeuplée, Tiers-monde affamé, Paris, Le Seuil, 1965, 313 p.

- DUMONT Réné, Chine, la Révolution culturale, Paris, Le Seuil, 1976, 220 p.

- FU TENG Margaret, «Les inégalités d'accès à l'enseignement primaire entre urbains et ruraux », Perspectives chinoises, $n^{\circ}$ 89, mai-juin, 2005, p. 30-36.

-HU Rong, « Economic Development and the Implementation of Village Elections in Rural China », Journal of Contemporary China, 14(44), août 2005, p. 427-444.

- HUANG Ping et Frank N. PIEKE, «China Migration Country Study », Conference on Migration Development and Pro-Poor Policy Choices in Asia, Dhaka, 22-24 juin 2003, p. 35-40.

- LAI Hairong, «Les élections semi-ouvertes de bourgs et de cantons au Sichuan », Perspectives chinoises, $\mathrm{n}^{\circ} 80$, novembre-décembre, 2003, p. 24.

- PONCET Sandra et Zhu NONG, « La dynamique migratoire des ruraux vers les villes », Perspectives chinoises, $\mathrm{n}^{\circ}$ 91, septembre-octobre 2005, p. 11-23.

- RocCA Jean-Louis, « Protestation sociale et coalition des élites - des réponses au vide institutionnel ?», Les Nouvelles de Chine, n 15, avril 2004, p. 17-21.

- ROULLEAU-BERGER Laurence et Shi LU, « Les travailleurs migrants à Shanghai. Inégalités, niches économiques et diversité des parcours d'accès à l'emploi », Perspectives chinoises, $\mathrm{n}^{\circ} 87$, janvierfévrier 2005, p. 2-9.

- SANjuAn Thierry, La Chine. Territoire et société, Paris, Hachette, 2000. 
- ZHANG Mei, China's Poor Regions. Rural-Urban Migration. Poverty, Economic Reform and Urbanisation, Londres et New York, Routledge Curzon, 2003.

- ZHENG Yongnian, «The New Policy Initia-tives in China's 11"t 5-Year Plan », Briefing Series - Issue 1, China Policy Institute, The University of Nottingham, $12 \mathrm{p}$.

\section{NOTES}

1. On trouvera des descriptions et des analyses détaillées de ces épisodes de l'histoire rurale chinoise dans Marie-Claire Bergère, La Chine de 1949 à nos jours, Paris, Armand Colin, 2000, et dans les ouvrages que leur a consacrés René Dumont: Révolution dans les campagnes chinoises, Paris, Le Seuil, 1957 ; La Chine surpeuplée, Tiers-monde affamé, Paris, Le Seuil, 1965 ; Chine, la Révolution culturale, Paris, Le Seuil, 1976.

2. Marie-Claire Bergère, op. cit., p. 174 ; Claude Aubert, Ying Cheng et Xiande Li, «Éthique et économie paysanne en Chine ", in Marc Dufumier (dir.), Un agronome dans son siècle, actualité de René Dumont, Paris, Karthala, 2002, p. 131-138 ; Thierry Sanjuan, La Chine. Territoire et société, Paris, Hachette, 2000, p. 65.

3. Ibid.

4. Thierry Sanjuan, op. cit., p. 144.

5. Claude Aubert (a), «Le devenir de l'économie paysanne en Chine », Revue Tiers-Monde, $\mathrm{n}^{\circ} 183$, juillet-septembre 2005, p. 15 de la version en ligne (http://www.ivry.inra.fr/mona/ publications_chercheurs/Textes-Publis/Aubert-TMChine.pdf)

6. Marie-Claire Bergère, op. cit., p. 175.

7. Claude Aubert (b), «Politiques agricoles chinoises : la porte étroite », in L'économie mondiale 2006, Paris, CEPI et La Découverte, coll. «Repères », 2005, p. III de la version en ligne (http:// www. ivry.inra.fr/mona/publications_chercheurs/Textes-Publis/Aubert-EcoMond06.pdf).

8. D'après certains observateurs, la prise en compte de ces différents facteurs extérieurs aux revenus donnerait plutôt un rapport de 5/1. Mei Zhang, China's Poor Regions. Rural-Urban Migration. Poverty, Economic Reform and Urbanisation, Londres et New York, Routledge Curzon, 2003, p. 34 .

9. Notons que les paysans chinois ont toujours été lourdement taxés. Ces taxes étaient composées de taxes agricoles et para-agricoles versées au district, des "prélèvements unifiés » (tongchou), taxes versées aux cantons, et des « retenues» (tiliu) versées au village. Jusqu'en 2004 s'ajoutait à cette série l'obligation pour les paysans de livrer des quotas de grains (essentiellement blé et riz) payés à prix administratifs bas. Ce «fardeau paysan » (nongmin fudan) aurait représenté en moyenne 10 à $15 \%$ du revenu net paysan, mais pouvait être plus élevé dans les districts pauvres, majoritairement agricoles, qui ne pouvaient pas compter sur la taxation des entreprises rurales par exemple. Certains districts des provinces de l'intérieur comme le Hubei et l'Anhui auraient affiché des taux réels d'imposition dépassant $25 \%$ du revenu net des paysans. Claude Aubert (a), art. cit., p. 18-19 de la version en ligne.

10. Ibid., p. 4 de la version en ligne.

11. Pour de plus amples précisions sur la question de l'éducation dans les campagnes chinoises, se référer à Margaret Fu Teng, «Les inégalités d'accès à l'enseignement primaire entre urbains et ruraux ", Perspectives chinoises, $n^{\circ} 89$, mai-juin, 2005, p. 30-36.

12. À ce titre l'été 2006 demeure exemplaire. Alors que les provinces du Fujian et du Guangdong ont été touchées par un violent typhon (le plus violent de ces cinquante dernières années d'après les médias chinois), la province du Sichuan a connu quant à elle une terrible sécheresse qui a dévasté de nombreuses récoltes. Entre mai et août, pas moins de vingt-sept provinces auraient subi des aléas climatiques touchant au total plus de 130 millions de personnes et en déplaçant 
3 millions. La Croix-Rouge chinoise aurait fourni une assistance dans dix-neuf provinces, durement touchées par des pluies torrentielles et des inondations, et parmi lesquelles figurent l'Anhui, le Guangxi, le Hunan, le Jiangxi et le Sichuan.

13. Claude Aubert (a), art. cit., p. 11 de la version en ligne.

14. Rappelons simplement qu'en décembre 2005 la police a ouvert le feu sur des manifestants à Dongzhou, un village de la province du Guangdong, faisant trois morts selon le bilan officiel. Toujours dans le Guangdong, en janvier 2006, d'autres émeutes se seraient soldées par la mort d'un enfant et une centaine de blessés.

15. Ces constatations sont issues d'une synthèse commentée par Jean-Louis Rocca (« Protestation sociale et coalition des élites - des réponses au vide institutionnel ? ", Les Nouvelles de Chine, $\mathrm{n}^{\circ} 15$, avril 2004, p. 17-21) d'un article de Chen Yingfang intitulé, «Enquête sur les canaux d'expression des intérêts des groupes indigents " (en chinois), Zhanlüe yu guanli, $\mathrm{n}^{\circ}$ 6, décembre 2003, p. 87-92.

16. Pour plus de précisions sur ces différents objectifs des années 2004 et 2005, se référer à Claude Aubert (b), art. cit., p. I-III de la version en ligne.

17. Ibid., p. $X$ de la version en ligne.

18. 18 - Claude Aubert (a), art. cit., p. 15 de la version en ligne.

19. Pour plus de précisions concernant les dynamiques migratoires et l'intégration des migrants dans le monde urbain, voir notamment : Sandra Poncet et Zhu Nong, «La dynamique migratoire des ruraux vers les villes", Perspectives chinoises, $n^{\circ}$ 91, septembre-octobre 2005, p. 11-23; Laurence Roulleau-Berger et Shi Lu, «Les travailleurs migrants à Shanghai. Inégalités, niches économiques et diversité des parcours d'accès à l'emploi ", Perspectives chinoises, $\mathrm{n}^{\circ} 87$, janvierfévrier 2005, p. 2-9.

20. 20 - Pour les ruraux, se rendre temporairement dans les aires urbaines sans un changement de registre n'était possible qu'avec l'autorisation des autorités locales et sous certaines conditions (visites de familles, contrat de travail, missions officielles).

21. Huang Ping et Frank N. Pieke, "China Migration Country Study», Conference on Migration Development and Pro-Poor Policy Choices in Asia, Dhaka, 22-24 juin, 2003, p. 35-40.

22. Les différentes réformes engagées dans les domaines de la santé et du logement, ainsi que la disparition progressive de la danwei (unité de travail) et les difficultés rencontrées par beaucoup d'entreprises d'État, ont de fait amenuisé, voire éliminé, un certain nombre de ces privilèges au cours des années 1990. Seul l'accès au système éducatif, obligatoire et gratuit jusqu'à la fin du lycée (gao zhongxue) pour les résidents urbains, est resté opérationnel.

23. Il s'agit notamment d'abolir les différentes mesures qui empêchaient jusque-là les migrants ruraux d'obtenir certains emplois permanents ou temporaires dans les zones urbaines, d'encourager un traitement des migrants identique à celui des citadins sur le marché urbain de l'emploi, de combattre les frais et renvois injustifiés, d'obliger les entreprises à signer des contrats de travail réglementaires et à verser les salaires de manière régulière, de permettre aux migrants un accès raisonnable aux soins de santé et à la sécurité sociale, ainsi que d'ouvrir les écoles urbaines à leurs enfants.

24. La municipalité de Shanghai a par exemple mis en place durant les années 1990 un type particulier de hukou urbain, appelé le "hukou bleu» (lan hukou), qu'elle vend aux migrants ruraux. Il permet de résider de manière permanente dans la ville et d'accéder à quelques services et privilèges spécialement accordés aux populations urbaines. Cependant, pour l'obtenir, des sommes considérables, représentant souvent plusieurs mois de salaires, sont nécessaires. Les autorités centrales se sont à plusieurs reprises élevées contre ce procédé.

25. Claude Aubert (b), art. cit., p. VII de la version en ligne.

26. Jie Chen, « Sociopolitical Attitudes of the Masses and Leaders in the Chinese Village : Attitude Congruence and Constraint ", Journal of Contemporary China, 14(44), August, 2005, p. 445-464. 
27. Weimin Cheng, "Some Thoughts on the Subjectivity of Villager's Self-government ", Social Sciences in China, hiver 2005, p. 26-30. Hairong Lai, « Les élections semi-ouvertes de bourgs et de cantons au Sichuan ", Perspectives chinoises, $n^{\circ}$ 80, novembre-décembre, 2003, p. 24.

28. Wei Ding, « Réforme des tribunaux de base et application de la loi dans la Chine rurale. Le cas de l'arrondissement de Weicheng, ville de Xianyang, province du Shaanxi », Perspectives chinoises, $\mathrm{n}^{\circ} 90$, juillet-août 2005, p. 45 et p. 47-48.

29. Hairong Lai, art. cit., p. 24.

30. Rong $\mathrm{Hu}$, «Economic Development and the Implementation of Village Elections in Rural China », Journal of Contemporary China, 14(44), août 2005, p. 427-444.

INDEX

Index géographique : Chine

Keywords : China, rural malaise, urban-rural inequalities, migration

Mots-clés : malaise rural, inégalités villes-campagnes, migration

\section{AUTEUR}

\section{SÉBASTIEN COLIN}

Docteur en géographie (spécialité géopolitique) de l'université Paris 8 , Sébastien Colin poursuit ses recherches sur la frontière sino-coréenne et sur les villes frontalières du Nord-Est chinois. Il assure des charges d'enseignement sur la géographie de la Chine, à l'INALCO et à l'université Paris 8, ainsi que sur la géopolitique des deux Corées à l'université de Marne-la-Vallée. Il a publié dans Perspectives chinoises, Hérodote, Images économiques du monde, Extrême-Orient/ Extrême Occident, Les études du CERI, ainsi que dans divers ouvrages collectifs, dont Asie orientale : intégrations régionales (dir. C. Taillard, 2004) et L'éveil du dragon (dir. F. Lasserre, 2005). Il a participé au Dictionnaire de la Chine contemporaine.

sebastien_colin@hotmail.com 